period after injury and was certainly the reason for the tissue losses seen in this brief study.

Enteral diets used with an intact gut are a useful form of nutritional support, and nutritional balance seems to be more easily achieved with a whole-protein diet. Nevertheless, enteral diets used in the period after injury require as much attention to detail as parenteral feeding if maximum benefit is to be obtained. Proprietary enteral diets are expensive in comparison with tube feeds prepared in hospital diet kitchens. The relative merits of these diets compared with the proprietary products used in this study have yet to be examined.

We are grateful to the consultant staff of the Regional Neurosurgical Centre for permission to study their patients, and gratefully acknowledge the invaluable help of the nursing staff.

Requests for reprints should be sent to Mr P D Wright.

\section{References}

${ }^{1}$ Silk DBA. Physiology of protein absorption. Research and Clinical Forums 1979;1:29-34.

${ }^{2}$ Matthews DM, Payne JW. Nutrition of micro organisms and animals. In: Matthews DM, Payne JW, eds. Peptide transport and protein nutrition. Amsterdam: Associated Scientific Publishers, 1975:1-60.

${ }^{3}$ Jelliffe DB. Assessment of the nutritional state of the community. WHO Monograph 53. Geneva: World Health Organisation, 1966.

4 Varley H. Practical clinical biochemistry. 5th ed. London: Heinemann, 1967:193.

${ }^{5}$ Baker SP, O'Neill B, Haddon W, Long WB. The injury severity score: a method for describing patients with multiple injuries and evaluating emergency care. 7 Trauma 1974;14:187-96.

- Elwyn DH, Gump FE, Iles M, Long CL, Kinney JM. Protein and energy sparing of glucose added in hypocaloric amounts to peripheral infusions of amino acids. Metabolism 1978;27:325-31.

(Accepted 10 March 1980)

\title{
Staphylococcal bacteraemia, fusidic acid, and jaundice
}

\author{
M W HUMBLE, SUSANNAH J EYKYN, I PHILLIPS
}

\section{Summary and conclusions}

Fusidic acid was used to treat 131 out of 250 patients with staphylococcal bacteraemia over 10 years. Other antimicrobial agents were given to the 119 remaining patients. Thirty-seven patients were already jaundiced before antibiotic treatment was started. Jaundice developed during treatment in 38 out of 112 patients given fusidic acid (34\%) and in two out of 101 patients given other antimicrobials. The incidence of jaundice was higher in patients given fusidic acid intravenously $(48 \%)$ rather than by mouth $(13 \%)$. Jaundice appeared within 48 hours after the administration of fusidic acid in $93 \%$ of these cases. When the drug was stopped serum bilirubin concentrations fell to normal values within four days in those patients in whom they had been previously normal and who survived the bacteraemic episode. Fusidic acid was associated with increasing jaundice in 13 of 19 patients $(68 \%)$ already jaundiced before it was given. In six out of 32 patients who developed jaundice while receiving intravenous fusidic acid serum alkaline phosphatase activity was raised suggestive of cholestatic jaundice. The mechanism in the remaining patients was unknown.

Fusidic acid, particularly the intravenous preparation, is invaluable in treating severe staphylococcal infection but should be used with caution in patients with abnormal liver function. Patients receiving intravenous fusidic acid should be given the oral form of the drug as soon as their clinical condition permits.

\section{Introduction}

Fusidic acid is effective in vitro against Staphylococcus aureus and in treating severe staphylococcal infection. ${ }^{1-4}$ Populations of most staphylococci sensitive to fusidic acid, however, contain

\footnotetext{
Department of Microbiology, St Thomas's Hospital Medical School, London SE1 7EH

$M W$ HUMBLE, MRCPATH, lecturer (present appointment: consultant microbiologist, Wellington Hospital, New Zealand)

SUSANNAH J EYKYN, MRCPATH, senior lecturer

I PHILLIPS, MD, MRCPATH, professor
}

a few resistant organisms, ${ }^{5}$ and fully resistant populations emerge rapidly after exposure to the drug both in vitro ${ }^{5}$ and in vivo $^{6}$; hence it is usually given in combination with another antimicrobial. ${ }^{6}$

In recent reviews of drug jaundice ${ }^{7}$ and drug-induced liver disease $^{8}$ fusidic acid was not mentioned. Ernst ${ }^{9}$ reported increased activities of serum aspartate transaminase and serum alanine transferase in four of 73 patients with chronic staphylococcal osteitis and osteomyelitis treated with fusidic acid, and one of these four developed persistent ascites and chronic hepatitis. Menday and March, ${ }^{10}$ in a review of 46 patients treated with intravenous fusidic acid for severe staphylococcal infection, reported on two who became progressively jaundiced during treatment. One was already jaundiced before the drug was given, and in the other, who developed overwhelming staphylococcal pneumonia after influenza, the jaundice was attributed to the toxaemic process of the disease. Copperman $^{11}$ treated three patients with intravenous fusidic acid for severe staphylococcal infection: one had jaundice before the drug was given, but this disappeared during treatment. A further case of jaundice possibly associated with fusidic acid was reported by Craig ${ }^{12}$ in a patient with acute pustular psoriasis, who was also given clindamycin. The jaundice occurred three days after the antibiotics were started, and the bilirubin concentration fell two days after they were stopped.

Because of a clinical impression that fusidic acid, especially the intravenous preparation, might be associated with jaundice, we investigated cases of staphylococcal bacteraemia seen at this hospital during the past 10 years.

\section{Patients and methods}

From October 1969 the medical staff of the microbiology department kept prospective records of all patients with bacteraemia Aspects that might have predisposed to jaundice were recorded, including pre-existing liver or biliary tract disease, concomitant administration of potentially hepatotoxic drugs, and recent major operations. Monitoring liver function tests in staphylococcal bacteraemia was not part of the prospective study, and we did not attempt to influence these investigations when the patient was in hospital, since we were not initially aware of any association between fusidic acid and jaundice. The liver function tests were thus performed when clinically indicated and analysed retrospectively. 
Three liver function tests were routinely performed by the department of clinical chemistry - namely, measurements of total serum bilirubin concentration, serum alkaline phosphatase activity, and serum aspartate transaminase activity. In three patients the conjugated and unconjugated serum bilirubin concentrations were measured.

\section{Results}

During the 10 years (1969-79) 250 episodes of staphylococcal bacteraemia occurred in 237 patients: fusidic acid was used in 131 episodes, being prescribed by the clinician usually in consultation with a microbiologist. No attempt was made to conduct a clinical trial, nor were patients specifically allocated to a particular treatment group. Table I shows the site of infection in patients with staphylococcal bacteraemia for all episodes in both treatment groups. Most patients receiving haemodialysis were treated with vancomycin, cloxacillin, or flucloxacillin, which explains the large number of these patients in the group not receiving fusidic acid. The average age of patients treated with fusidic acid was 41 years (range six weeks to 76 years) and that of patients treated with other antimicrobials 45 years (range four days to 85 years).

TABLE I-Site of infection in 250 episodes of staphylococcal bacteraemia in patients treated with fusidic acid or other antimicrobials

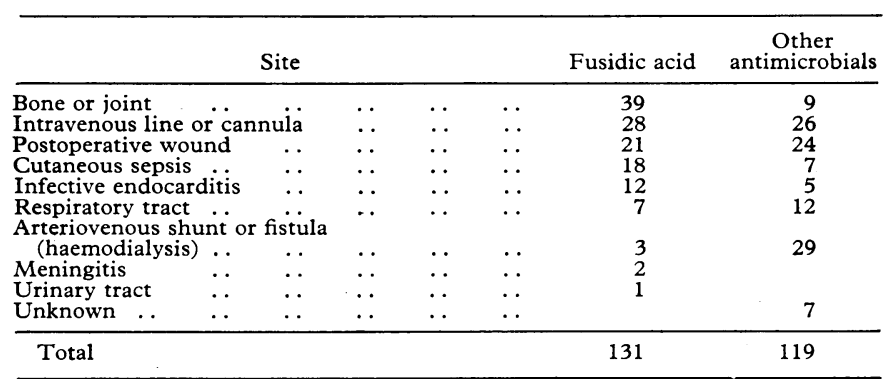

\section{ANTIBIOTIC REGIMENS}

Fusidic acid was used in 131 bacteraemic episodes: it was given intravenously in 43 , by mouth in 38 , and by both routes in 50 . In general, those patients given intravenous fusidic acid were more seriously ill than those treated with the oral preparation. The drug was given intravenously for an average of seven days (range 1-42 days) and by mouth for an average of 28 days (range 2-154 days). The intravenous preparation was infused over two to four hours, in a dose in adults of $500 \mathrm{mg}$ eight hourly. The dosage regimen in children was that recommended by the manufacturers-namely, $20-40 \mathrm{mg} / \mathrm{kg}$ body weight daily in three equal doses. Fusidic acid was always given with a second antimicrobial, and table II shows the drugs used together with the drugs used in the patients not treated with fusidic acid.

TABLE II-Antimicrobials used in treating staphylococcal bacteraemia

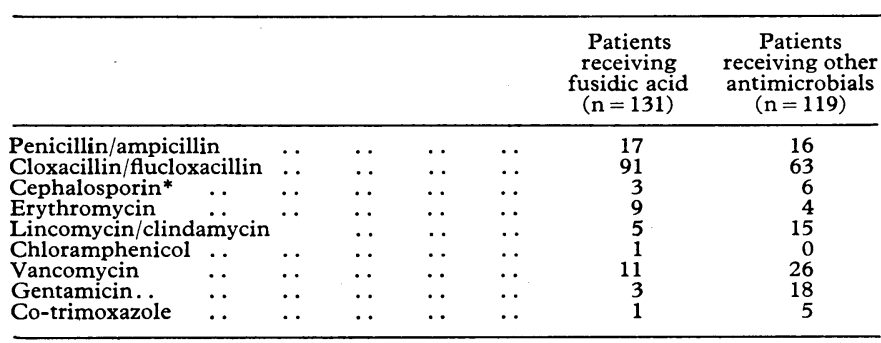

*Cephalothin five patients, cephaloridine two, cefazolin one, and cefuroxime one.

\section{LIVER FUNCTION}

Liver function tests were done in 91 of the 131 patients treated with fusidic acid and in 48 of the 119 patients who received other antimicrobials.

\section{BILIRUBIN CONCENTRATIONS}

Of the 131 patients given fusidic acid, 19 were jaundiced before treatment, and in 13 of these (nine of whom received the drug intravenously), the bilirubin concentration increased further during treatment (figure). When the fusidic acid was stopped the concentra-

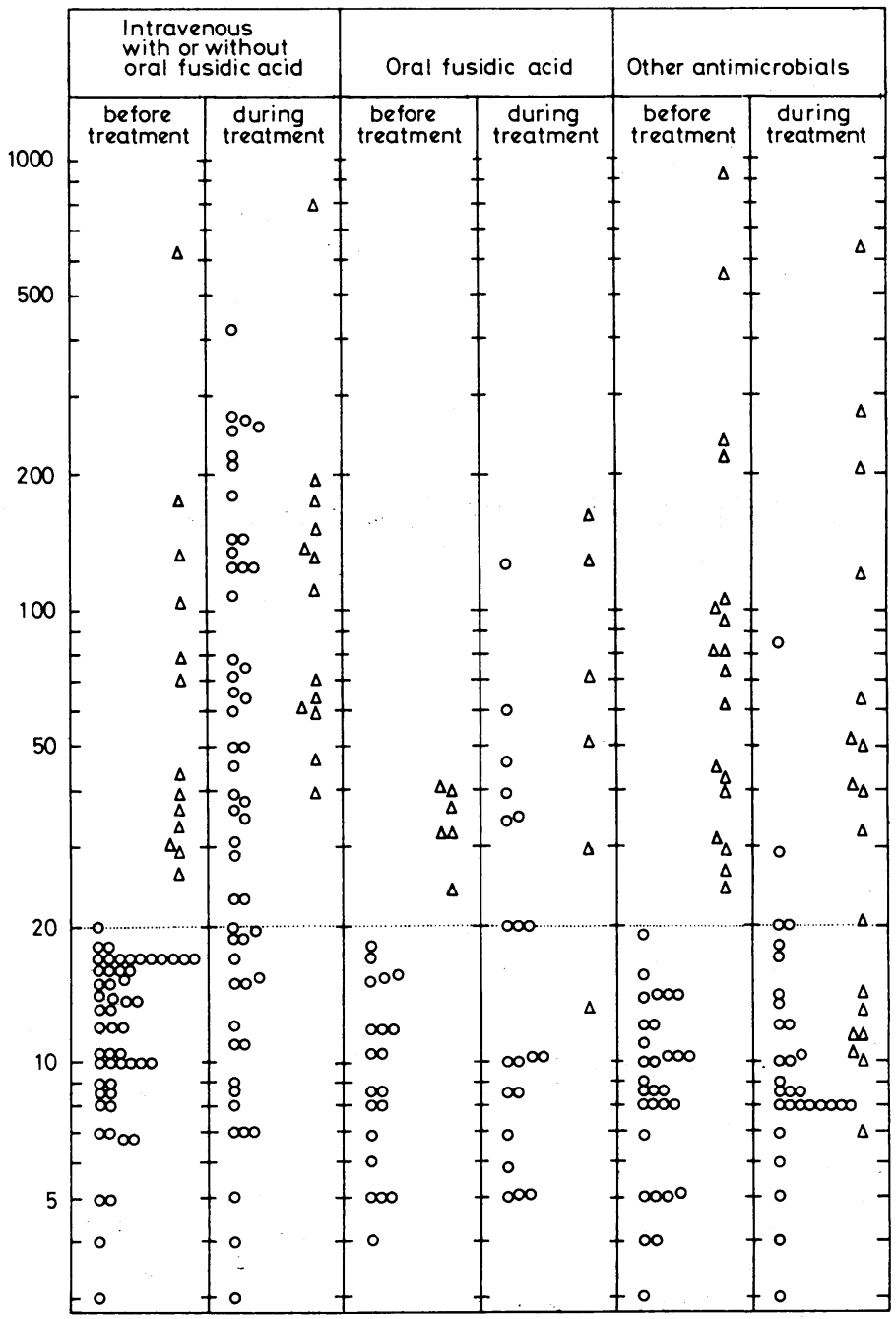

Maximum serum total bilirubin concentration recorded in 139 patients with staphylococcal bacteraemia before and during treatment with intravenous fusidic acid alone or with the oral preparation, oral fusidic acid alone, or other antimicrobial agents. (Normal laboratory reference values in range 5-20 mmol/1; 292-1169 mg/100 ml.)

$\mathrm{O}=$ Concentration normal before antimicrobials were given. $\triangle=$ Concentrations abnormal before antimicrobials were given.

Conversion: SI to traditional units-Bilirubin: $1 \mathrm{mmol} / 1 \approx 58.5 \mathrm{mg} /$ $100 \mathrm{ml}$.

tion fell in all patients who survived the bacteraemic episode, eventually reaching normal in five of the 13. Liver function was tested before treatment in 72 of the 112 remaining patients: the bilirubin concentration was normal in all cases. The 40 patients not tested were not clinically jaundiced. Thirty-eight of the 112 patients developed jaundice during treatment with fusidic acid (table III, figure)-namely, 32 of the $66(48 \%)$ given the intravenous preparation, with or without the oral preparation, and six of the $46(13 \%)$ given only the oral preparation $\left(\chi^{2}=13 \cdot 7, p<0.0005\right)$. Out of 25 patients treated with both oral and intravenous fusidic acid, 10 developed jaundice during the period of intravenous administration: in six of these the bilirubin concentration fell to normal when the oral preparation was substituted, whereas in the others it continued to rise.

Bilirubin was largely conjugated in the three patients in whom conjugated and unconjugated concentrations were measured. The increase in bilirubin concentration occurred during the first 48 hours of treatment with intravenous fusidic acid in most patients, reaching 
TABLE III-Results of liver function tests before and during treatment in patients who developed jaundice during treatment

\begin{tabular}{|c|c|c|c|c|c|c|c|c|}
\hline & \multicolumn{4}{|c|}{ Fusidic acid $(n=38)$} & \multicolumn{4}{|c|}{ Other antimicrobials $(n=2)$} \\
\hline & Normal & $\underset{\text { raised }}{\mathrm{AP}}$ & $\underset{\text { raised }}{\mathrm{AT}}$ & $\begin{array}{c}\mathrm{AP} \text { and } \\
\mathrm{AT} \\
\text { raised }\end{array}$ & Normal & $\underset{\text { raised }}{\text { AP }}$ & $\underset{\text { raised }}{\mathrm{AT}}$ & $\begin{array}{c}\mathrm{AP} \text { and } \\
\mathrm{AT} \\
\text { raised }\end{array}$ \\
\hline \multirow{5}{*}{$\begin{array}{l}\text { Before treatment } \\
\text { During treatment: } \\
\text { Bilirubin raised } \\
\text { Bilirubin and AP } \\
\text { raised } \\
\text { Bilirubin and AT } \\
\text { raised } \\
\text { Bilirubin, AP, and } \\
\text { AT raised }\end{array}$} & 17 & 7 & 9 & 5 & & & 1 & 1 \\
\hline & 7 & 5 & 6 & 3 & & & 1 & 1 \\
\hline & $\Gamma$ & & & & & & & \\
\hline & a & 1 & 2 & 2 & & & & \\
\hline & 4 & 1 & 1 & & & & & \\
\hline
\end{tabular}

$\mathrm{AP}=$ Alkaline phosphatase. $\mathrm{AT}=$ Aspartate transaminase

a maximum after about five days. In contrast, with oral fusidic acid the bilirubin concentration reached a maximum after about seven days. Intravenous fusidic acid was stopped because of increasing jaundice in 11 patients, and the bilirubin concentration fell to normal after about four days in those who survived the bacteraemic episode. In one patient the bilirubin concentration returned to normal while the antibiotic was still being given intravenously. There was no significant difference in the incidence of jaundice between the antibiotics used with fusidic acid: cloxacillin, flucloxacillin, or penicillin was given to 30 of the 38 patients who became jaundiced while receiving fusidic acid and to 65 of the 74 who did not.

Of the 119 patients who did not receive fusidic acid, 18 were already jaundiced before antimicrobial treatment began but no further increase in bilirubin concentration occurred. Liver function tests were done in 30 of the remaining patients: two of these developed jaundice, both while receiving intravenous clindamycin (table III). The difference in the incidence of jaundice between the patients given fusidic acid and those given other antimicrobials was highly significant $\left(x^{2}=33 \cdot 5, \mathrm{p}<0 \cdot 0001\right)$.

\section{ALKALINE PHOSPHATASE}

Serum alkaline phosphatase activity was measured in 91 patients who received fusidic acid; it was normal before treatment in 59 (eight already jaundiced) and became abnormal during treatment in 12 (two already jaundiced), and was raised before treatment in 32 (11 already jaundiced) and increased further during treatment in four (two already jaundiced). There was no significant difference in enzyme activity between patients given intravenous or oral fusidic acid. Serum alkaline phosphatase activity was measured in 48 of the patients treated with other antimicrobials: it was normal before treatment in 31 (nine already jaundiced) and increased during treatment in two (one already jaundiced), and was abnormal before treatment in 17 (nine already jaundiced) but did not increase further.

\section{ASPARTATE TRANSAMINASE}

Serum aspartate transaminase activity was measured in 91 patients who were treated with fusidic acid: it was normal before treatment in 53 (four already jaundiced) and increased during treatment in 16 (one already jaundiced), and was raised before treatment in 38 (15 already jaundiced) and increased further during treatment in four (none already jaundiced). There was no significant difference in enzyme activity between patients given intravenous or oral fusidic acid. Serum aspartate transaminase activity was measured in 48 of the patients given other antimicrobials: it was normal before treatment in 22 (four already jaundiced) and rose during treatment in four (one already jaundiced), and was abnormal before treatment in 26 (14 already jaundiced) and increased further during treatment in two (both already jaundiced).

\section{PREDISPOSING FACTORS}

There was no evidence to suggest that operations or using potentially hepatotoxic drugs predisposed to jaundice during treatment with fusidic acid, since these were equally common in patients who did not become jaundiced. Only one out of 112 patients not jaundiced before fusidic acid was given had known liver or biliary tract disease he became jaundiced while receiving intravenous fusidic acid 10 days after cholecystectomy for chronic cholecystitis. Of 19 patients already jaundiced before fusidic acid was given, 11 had had major operations and seven became increasingly jaundiced during treatment. One of these 19 was receiving steroids and became more jaundiced during treatment. Four of the 19 had pre-existing liver or biliary tract disease, and two became more jaundiced while receiving fusidic acid. Of 18 patients already jaundiced but not given fusidic acid, seven had had operations, three were receiving steroids, one was receiving cytotoxic drugs, and three had pre-existing liver or biliary tract disease.

\section{OUTCOME}

Of the 112 patients not jaundiced before fusidic acid was given, 26 died $(23 \%)$, of whom $19(73 \%)$ were jaundiced at death. Serum alkaline phosphatase activity was raised in eight of these 19 patients, but in no case above $50 \mathrm{KA}$ units/dl. Serum aspartate transaminase activity exceeded $200 \mathrm{IU} / 1$ in three patients, in only one of whom was alkaline phosphatase activity raised. Death was related to staphylococcal bacteraemia in nine of the 19 jaundiced patients and unrelated to either staphylococcal bacteraemia or liver failure in 10 . Of the 86 patients given fusidic acid who survived, $17(20 \%)$ were jaundiced.

Of the 101 patients not jaundiced who received antimicrobials other than fusidic acid, 25 died (only one jaundiced at death). Of the 76 patients who survived, a further one was jaundiced.

Nineteen patients were already jaundiced before fusidic acid was given, of whom seven died $(37 \%)$. Death was related to bacteraemia in three of these patients. Eighteen patients were already jaundiced before other antimicrobials were given and six died, all jaundiced at death. Death was related to the bacteraemia in three.

\section{Discussion}

Studying drug-associated jaundice in patients with staphylococcal bacteraemia is complicated because the infection may be accompanied by jaundice. ${ }^{13}$ This study enabled us to investigate the effect of fusidic acid on liver function in patients with staphylococcal bacteraemia by comparing groups of patients treated with fusidic acid and other antimicrobials. It shows convincingly that the incidence of reversible jaundice is increased in patients treated with intravenous fusidic acid. We have no evidence that the patients who received intravenous fusidic acid were more seriously ill and thus more likely to become jaundiced than those receiving other intravenous antimicrobials. Although there was no significant difference in mortality between the patients given fusidic acid and those who received other antimicrobials, the incidence of jaundice was far higher in patients who died while receiving fusidic acid. We have no evidence, however, that the jaundice associated with fusidic acid caused the death of any patient.

The mechanism of the jaundice associated with fusidic acid is not known: experimental evidence (data on file, Leo Laboratories) suggests that the intravenous preparation can cause haemolysis, depending on the concentration and rate of the infusion. Parsons et al, ${ }^{14}$ however, gave $250 \mathrm{mg}$ of fusidic acid intravenously over 15 minutes, six hourly for four days, to a child without producing haemolysis. They also gave $500 \mathrm{mg}$ of fusidic acid intravenously over 15 minutes to eight normal subjects, again without haemolysis. Single dosing in volunteers may not be comparable with repeated dosing in patients with septicaemia, but there was no gross evidence of haemolysis in any of our patients, nor did any of them receive the infusion over only 15 minutes. We conclude that acute haemolysis is unlikely to have been responsible for the jaundice observed in our patients. An alternative explanation may lie in the steroidlike structure of fusidic acid, ${ }^{15}$ since steroids and steroid-related drugs may cause cholestasis and jaundice. ${ }^{6}{ }^{7}$

Fusidic acid is concentrated in the bile, ${ }^{15}$ and one of its principal metabolites is a complex of fusidic acid and glucuronic acid. Experiments in rat liver slices (data on file, Leo Laboratories) have shown that a concentration of $500 \mathrm{mg}$ 
fusidic acid/l inhibits the excretion of conjugated bilirubin, and at $3000-5000 \mathrm{mg} / \mathrm{l}$ there is competitive inhibition of conjugation. These concentrations are considerably greater than any likely to have been reached in our patients. In six out of 32 patients who became jaundiced during treatment with intravenous fusidic acid, however, the serum alkaline phosphatase activity increased; and the bilirubin was predominantly conjugated in the three patients in whom conjugated and unconjugated concentrations were measured. These findings may indicate that the jaundice was cholestatic in a few cases. For the rest we have no evidence for such a mechanism. A careful prospective investigation would be needed to elucidate the mechanism of the jaundice associated with fusidic acid treatment.

Since the jaundice is reversible and the clinical results obtained with fusidic acid in severe staphylococcal sepsis are excellent, the continuing use of this valuable agent would seem to be justified. We make the following suggestions. Firstly, the intravenous preparation should be made up in the volume recommended by the manufacturer $(500 \mathrm{ml})$ or in the maximum volume compatible with the patient's clinical condition and infused slowly over at least six to eight hours. Secondly, as soon as the patient's condition permits the oral form of the drug should be used. Thirdly, liver function should be monitored regularly in any patient receiving intravenous fusidic acid. Should the bilirubin concentration continue to rise when the patient is given oral fusidic acid the drug should be stopped. In patients already jaundiced a further increase in bilirubin concentration during treatment with fusidic acid is also an indication for stopping the drug, although the cause of the deterioration in liver function can seldom be determined.

We thank the many doctors at St Thomas's Hospital for permission to study their patients; and Dr B Slavin, Miss P Kind, the staff of the department of clinical chemistry, Dr R Pounder, and Mr A P Menday (Leo Laboratories) for help and advice.

Requests for reprints should be sent to Professor I Phillips.

\section{References}

1 Jensen K, Lassen HCA. Fulminating staphylococcal infections treated with Fucidin and penicillin or semi-synthetic penicillin. Ann Intern Med $1964 ; 60: 790-8$.

2 Jensen K, Lassen HCA. Combined treatment with antibacterial chemotherapeutic agents in staphylococcal infections. $Q \mathcal{F}$ Med 1969;38: 91-106.

3 Blockey NJ, McAllister TA. Antibiotics in acute osteomyelitis in children. f Bone foint Surg 1972;54B:299-309.

4 Wright GLT, Harper J. Fusidic acid and lincomycin therapy in staphylococcal infections in cystic fibrosis. Lancet 1970;i :9-14.

5 Barber M, Waterworth PM. Antibacterial activity in vitro of Fucidin. Lancet $1962 ; \mathrm{i}: 931-2$.

${ }^{6}$ Lowbury EJL, Cason JS, Jackson DMacG, Miller RWS. Fucidin for staphylococcal infection of burns. Lancet $1962 ; \mathrm{ii}: 478-80$.

7 Paton A. Drug jaundice. Br Med f 1976;ii:1126-7.

${ }^{8}$ Keeling PWN, Thompson RPH. Drug-induced liver disease. Br Med $\mathcal{F}$ $1979 ; \mathrm{i}: 990-3$.

${ }^{9}$ Ernst J. Fucidin behandlung ved kroniske stafylokokostitter og osteomyelitter. Ugeskr Laeger 1969;131:779-83.

10 Menday AP, March BT. Intravenous fusidic acid in the management of severe staphylococcal infections: a review of 46 cases. Curr Med Res Opin 1976;4:132-8.

11 Copperman IJ. The prolonged use of intravenous fusidic acid in severe staphylococcal infection. Br f Clin Pract 1972;26:83-4.

12 Craig JA. Jaundice in acute pustular psoriasis. Br Med $\mathcal{F} 1974 ;$ iii 43.

${ }^{13}$ Fahrlander H, Huber F, Gloor F. Intra-hepatic retention of bile in severe bacterial infections. Gastroenterology 1964;47:590-9.

14 Parsons RL, Beavis JP, Paddock GM, David JA, Trounce JR. Additives to intravenous fluids. $B r$ Med $\mathcal{F} 1976$;iv: 1256 .

15 Godtfredsen WO, Vangedal S. On the metabolism of fusidic acid in man. Acta Chem Scand 1966;20:1599-607.

(Accepted 11 March 1980)

\title{
Adverse reactions to D-penicillamine after gold toxicity
}

\author{
M J DODD, I D GRIFFITHS, M THOMPSON
}

\section{Summary and conclusions}

The incidence of adverse reactions to $D$-penicillamine in 155 patients with rheumatoid arthritis was analysed and compared with their history of adverse reactions to gold. Out of 125 patients who took only D-penicillamine, 45 developed side effects from the drug, whereas of 27 patients with a history of gold toxicity, 18 also reacted adversely to D-penicillamine. All patients who took $\mathrm{D}$-penicillamine within six months after an adverse reaction to gold developed side effects from D-penicillamine. Fourteen patients developed similar adverse reactions to $D$-penicillamine and gold, and the interval between treatments in this group was significantly shorter $(p<0.01)$ than in those who developed either differing adverse reactions to both drugs or no reaction to $\mathrm{D}$-penicillamine after treatment with gold.

An interval exceeding six months between treatment with gold and treatment with $D$-penicillamine in patients who have developed adverse reactions to gold apparently reduces the risk of adverse reactions to $D$-penicillamine.

\footnotetext{
Department of Rheumatology, Freeman Hospital, Newcastle upon Tyne NE1 4LP

M J DODD, MB, MRCP, senior registrar

I D GRIFFITHS, MB, MRCP, consultant rheumatologist

M THOMPSON, MD, FRCP, consultant rheumatologist
}

\section{Introduction}

Both gold salts and D-penicillamine are effective against rheumatoid arthritis, and the therapeutic responses to the two drugs are apparently similar. ${ }^{1}$ Although both drugs produce a high incidence of adverse reactions, ${ }^{23}$ it has not been clearly established whether patients reacting adversely to gold are more likely to develop adverse reactions to D-penicillamine.

Day and Golding ${ }^{4}$ noted that three out of four patients who developed bone marrow depression while taking D-penicillamine had already taken gold, and a later report ${ }^{5}$ implied that rashes and blood dyscrasias were more common in patients taking D-penicillamine who had already taken gold. A multicentre trial group, however, failed to show any association between the two drugs in terms of adverse reactions. ${ }^{\circ}$

We have analysed retrospectively the incidence of adverse reactions in patients treated with $\mathrm{D}$-penicillamine and related these findings to their history of adverse reactions to gold.

\section{Patients, methods, and results}

One hundred and fifty-five patients took D-penicillamine for rheumatoid arthritis, 30 of whom had already been treated with gold (sodium aurothiomalate), which was stopped because of either adverse reactions $(n=27)$ or lack of benefit $(n=3)$. Eighteen of the 27 patients $(67 \%)$ who stopped taking gold because of side effects also reacted adversely to $D$-penicillamine necessitating withdrawal of 